\title{
Giant melanotic neuroectodermal tumor of infancy (melanotic progonoma) of the head and neck: report of a malignant case
}

\author{
Giancarlo Nicosia, MD,,7 Pietro Spennato, MD, ${ }^{1}$ Ferdinando Aliberti, MD,1 \\ Daniele Cascone, MD, ${ }^{2}$ Lucia Quaglietta, MD, ${ }^{3}$ Maria Elena Errico, MD, ${ }^{4}$ Mario Muto, MD, ${ }^{5}$ \\ Franco lonna, MD, ${ }^{6}$ and Giuseppe Cinalli, MD ${ }^{1}$
}

\begin{abstract}
Departments of ${ }^{1}$ Paediatric Neurosurgery, ${ }^{2}$ Neuroradiology, ${ }^{3}$ Oncology, and ${ }^{4}$ Pathology, Santobono-Pausilipon Children's Hospital; ${ }^{5}$ Department of Neuroradiology, Antonio Cardarelli Hospital; ${ }^{6}$ Division of Maxillo-Facial and ENT Oncologic Surgery, Istituto Nazionale dei Tumori, IRCCS Fondazione Pascale, Naples; and 'Department of Neurosurgery, University of L'Aquila, Italy
\end{abstract}

\begin{abstract}
Melanotic neuroectodermal tumor of infancy is a rare congenital pigmented neoplasm of neural crest origin, locally aggressive, growing rapidly and developing during the 1st year of life. It most commonly arises from the maxilla, cranial vault, and mandible. Occasionally, it exhibits malignant behavior with local lymph nodes involvement. Cases misdiagnosed and left untreated for a long time can present challenges due to the tumor mass and infiltration. In these cases, adjuvant chemotherapy can be extremely helpful before radical excision.

Authors of this report describe a 4-year-old boy from a developing country who was referred to their hospital with an ulcerated bulging lesion in the midline/right parietooccipital region, extending to the right laterocervical and parotid regions, resulting in significant craniofacial deformation. Magnetic resonance imaging of the brain revealed a highly enhancing tumor with intracranial and extracranial development extending mainly at the level of the right parietooccipital region, with lytic and hypertrophic alterations of the skull. The patient was managed with neoadjuvant and adjuvant chemotherapy and radically resective surgery on metastatic lymph nodes and the primary tumor of the skull. Scheduled radiotherapy was not performed, according to the parents' wishes. The patient returned to his native country where the lesion recurred, and he ultimately died approximately 10 months after the end of the treatment.
\end{abstract}

The literature indicates that tumor removal alone has been the treatment of choice in most isolated cases, but in cases of highly advanced tumor with involvement of the skull and cervical lymph nodes, it is preferable to proceed with preoperative chemotherapy with the aim of reducing the tumor volume, allowing better technical conditions for complete surgical removal, and decreasing the risk of local recurrence or metastasis.

https://thejns.org/doi/abs/10.3171/2016.11.PEDS16509

KEY WORDS MNTI; melanotic neuroectodermal tumor of infancy; melanotic progonoma; chemotherapy; skull; preoperative embolization; endovascular treatment; oncology

$\mathrm{M}$ ELANOTIC neuroectodermal tumor of infancy (MNTI), also known as "melanotic progonoma," is a rare osteolytic pigmented lesion of neural crest origin, occurring in infants. ${ }^{1,20}$ Although most cases occur within the 1st year of life without any sex predilection, $, 2,1,15,18$ rare cases in older children and adults have been reported. It usually presents in the head and neck region:
$70 \%$ of cases occur in the maxilla, followed by the skull (11\%) and mandible (6\%)., ${ }^{5,12,17}$ It has also been documented in the extracranial sites such as the epididymis, mediastinum, and female reproductive organs.,12,14,15 In the skull, MNTI usually arises at the sutures, and about half of the cases occur around the anterior fontanelle. Brain parenchymal involvement generally results from intraaxial ex-

ABBREVIATIONS HVA = homovanillic acid; MNTI = melanotic neuroectodermal tumor of infancy; VMA = vanillyImandelic acid.

SUBMITTED September 5, 2016. ACCEPTED November 30, 2016.

INCLUDE WHEN CITING Published online February 24, 2017; DOI: 10.3171/2016.11.PEDS16509. 
tension of skull lesions, although cases of primary tumors arising in the cerebellar vermis and third ventricle have been reported. ${ }^{8}$

Patients typically present with a nontender, nonulcerated, rapidly growing soft tissue swelling that results in skull deformation. The tumor causes compression rather than infiltration of adjacent structures, with local invasion causing bony destruction, tooth displacement, and feeding difficulties. Although MNTI is mostly benign, a malignancy rate of $6.6 \%$ has been reported. ${ }^{15,20}$ Postoperative recurrence rates for MNTI range from $10 \%$ to $60 \% .{ }^{11}$ Late recurrence following resection is rare; however, $39.3 \%$ of all cases occur within 4 weeks of surgery and up to $71.4 \%$ of cases recur within 4 months. In cases in which surgery might be mutilating, chemotherapy has occasionally been successful.

We present the case of a giant malignant MNTI involving the whole occipitoparietal calvaria and the cervical lymph nodes bilaterally, treated with multistep surgery and pre- and postsurgical chemotherapy. We also review the literature concerning this type of tumor arising in the skull and discuss methods of treatment.

\section{Case Report}

\section{History and Examination}

A 4-year-old child from a developing country was referred to our hospital with significant craniofacial deformation produced by a very large ulcerated lesion bulging in the midline/right parietooccipital region and extending to the right laterocervical and parotid regions (Fig. 1A). His clinical history was unremarkable. His uncle, the only relative accompanying the boy, reported progressive growth of "a tumour in his head" beginning from the 2nd year of life. On admission, the patient had normal developmental milestones. He could not fully control movements of his head, which was tilted to one side, mainly because of the very large mass involving the head and neck. The ulcerated bulging lesion was firm, nonmobile, painless, and apparently adherent to deeper structures. The child had severe pain mainly due to severe swelling of the cervical lymph nodes, refusing any contact or manipulation.

Computed tomography study revealed a huge parietooccipital mass lesion with periosteal sclerosis, growing intracranially as well as extracranially with mass effect and massive bilateral involvement of the cervical lymph nodes. Magnetic resonance imaging revealed an expansile extraaxial mass with mixed signal intensity on T1- and $\mathrm{T} 2$-weighted sequences, hypointense signal on $\mathrm{T} 2 *$ sequences, and intense peripheral contrast enhancement mainly extending at the level of the right parietooccipital region; the right extracranial neoplastic portion invaded the parotid region and the parapharyngeal and retromandibular space, with compression of the neck vessels. Bilateral extracranial lymph node swellings at the laterocervical regions were also very significant (Fig. 2A-D). The trachea was severely deviated and compressed.

To obtain tissue sample for diagnosis, the patient underwent biopsy of a large metastatic left laterocervical lymph node while under general anesthesia. During this same session, tracheostomy was performed to avoid sudden respiratory distress and facilitate all procedures to come, and a central venous catheter was implanted. The immediate follow-up was uneventful and the patient came back on the ward after 1 night in the intensive care unit.

Histological evaluation revealed a biphasic neoplasm displaying nests of poorly differentiated neuroblast-like cells positive for synaptophysin and pigmented cuboidal epithelioid cell positive for cytokeratin, epithelial membrane antigen, and HMB-45 (Fig. 3 upper). In addition, some neoplastic cells were immunoreactive for synaptophysin as well as HMB-45 and epithelial markers (Fig. 3 lower). Several mitoses and apoptotic bodies were observed. The overall histological features were consistent with a rare malignant variant of MNTI.

Blood tests showed a high serum level of catecholamines and increased urinary excretion of vanillylmandelic acid (VMA) and homovanillic acid (HVA).

Because of the tumor's size as well as its intra- and extracranial involvement, age of the patient, involvement of the cervical lymph nodes, and very poor general conditions, immediate surgical treatment was not considered.

\section{Initial Treatment}

The patient was referred to oncologists to start chemotherapy and to a plastic surgeon for the cutaneous lesions. Curettage of the ulcerated skin and removal of the necrotic
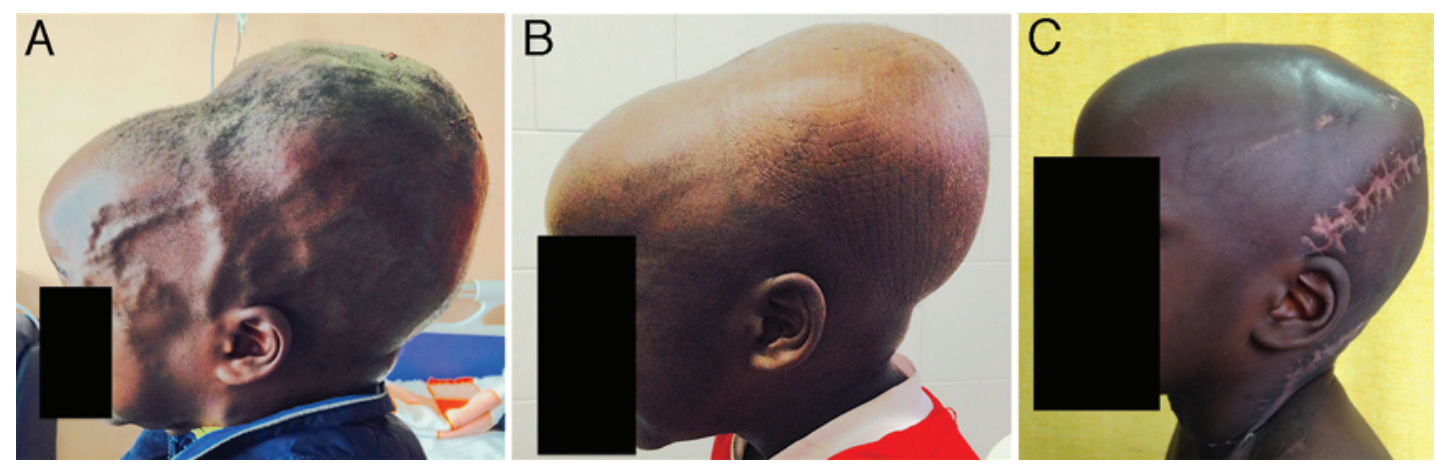

FIG. 1. A: External appearance of the patient at the time of admission, with a very large mass in the occipitoparietal region and significant enlargement of the subcutaneous venous system. B: Four months after admission, after 4 cycles of chemotherapy. C: Eight months after admission, 2 months following tumor removal and cranial vault reconstruction. Figure is available in color online only. 

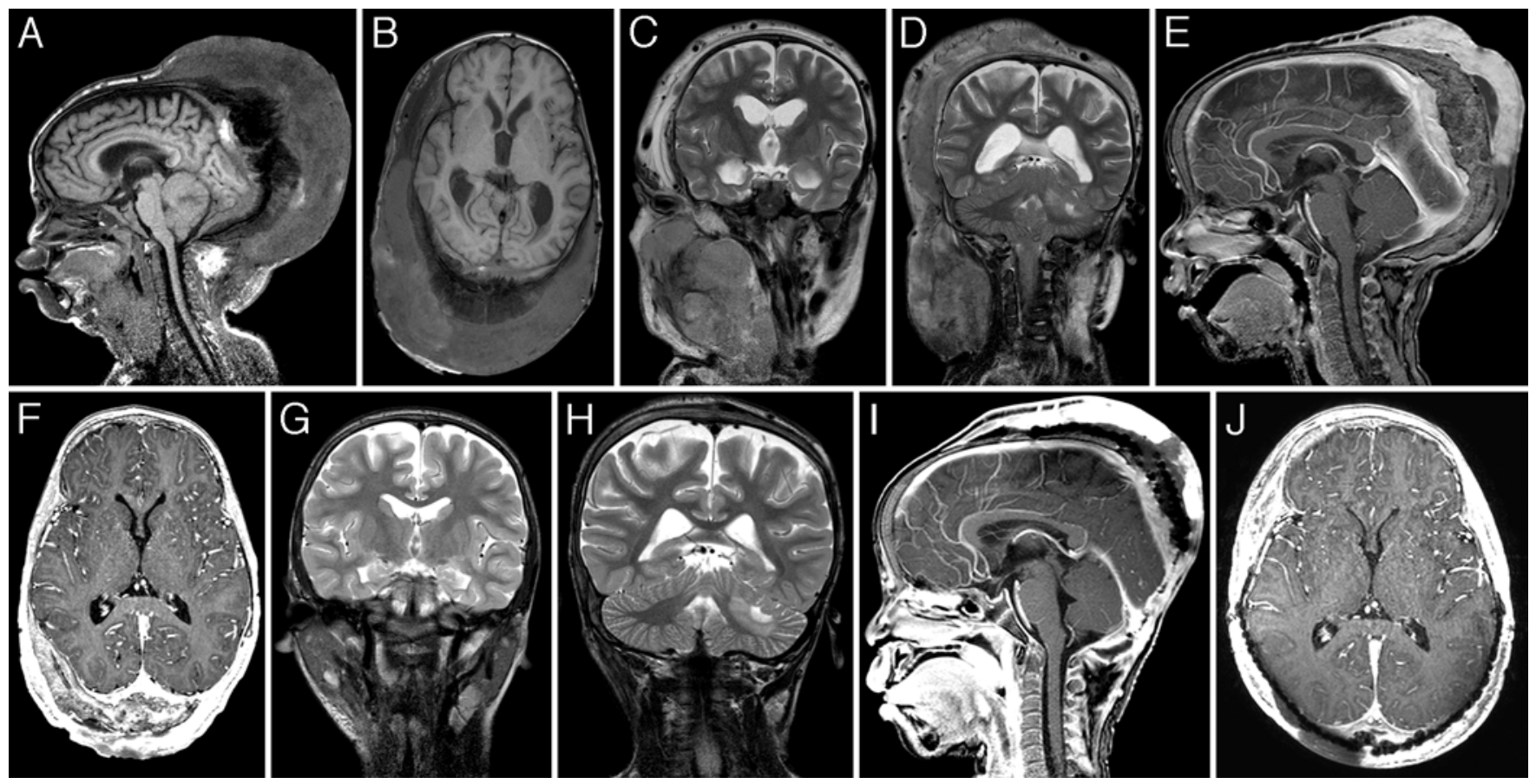

FIG. 2. Magnetic resonance imaging studies. Impressive calvarial thickening and very significant swelling of subcutaneous tissue of the neck and parietooccipital scalp are evident (A and $\mathbf{B})$. The subcutaneous mass is fused with significant swelling of the cervical lymph nodes that displaces the cervical vessels, larynx, and trachea ( $\mathbf{C}$ and $\mathbf{D})$. After 5 cycles of chemotherapy, calvarial thickening and subcutaneous infiltration are significantly decreased ( $\mathbf{E}$ and $\mathbf{F})$, as is cervical lymph nodes involvement ( $\mathbf{G}$ and $\mathbf{H})$. After 2 surgical procedures, the whole parietooccipital calvaria has been removed, except for a thin layer on the midline (I), and temporarily reconstructed with titanium meshes $(\mathrm{J})$.

tissue in the parietooccipital region allowed for slow healing of the cutaneous lesion. In the same time period, the patient started chemotherapy. He underwent 5 cycles of a combination of topotecan $\left(0.375 \mathrm{mg} / \mathrm{m}^{2}\right.$ at Days $\left.1-5\right)$ and cyclosphosphamide (125 mg/m² at Days 1-5), alternating with cyclosphosphamide $\left(4 \mathrm{~g} / \mathrm{m}^{2}\right.$ at Day 1$)$ and etoposide $\left(200 \mathrm{mg} / \mathrm{m}^{2}\right.$ at Days 2-4) or ifosfamide $\left(3 \mathrm{~g} / \mathrm{m}^{2}\right.$ at Days $1-3)$ and etoposide $\left(150 \mathrm{mg} / \mathrm{m}^{2}\right.$ at Days $\left.1-3\right)$, resulting in significant tumor reduction both on MR images and on external appearance (Figs. 1A-B and 2E-H). Blood tests during follow-up showed a rapid decrease in the high serum level of catecholamines.

Between the third and fourth chemotherapy cycle, a bilateral neck lymphadenectomy was performed. Histological analysis showed that 13 lymph nodes of the right lateral cervical chain, 9 lymph nodes of the left occipital cervical chain, 1 lymph node of the left lateral cervical chain (Level IV), and the right salivary gland were positive for metastases of MNTI. Immunohistochemical investigations confirmed histological analysis by showing immunoreactivity of some neoplastic cells for synaptophysin (Fig. 4).

Mass effect of the tumor on the upper airways markedly improved; therefore, the tracheostomy tube was removed.

Five months after the start of chemotherapy, surgery on the skull lesion was planned since the maximum point of chemotherapeutic effectiveness had been attained. Surgery at this stage was considered the only option for achieving complete removal while verifying the degree of soft tissue infiltration. If this had been deemed impossible, massive surgical debulking would have permitted the best cytoreductive effect before radiotherapy.

The patient underwent preoperative angiography with embolization because of the very impressive collateral circulation visible both at the physical examination (Fig. 1) and on MRI (Fig. 2), suggesting a highly vascularized lesion. Angiography showed that the extraaxial bulky tumor mass was primarily vascularized by branches of the external carotid arteries of both sides. Through a microcatheter positioned in the posterior branch of the superficial temporal artery and occipital branch, polyvinyl alcohol microparticles (diameter about 300-500 $\mu \mathrm{m}$, Contour SE, Boston Scientific Corp.) were injected. Occlusion of the arterial supply was intended purely as a presurgical step to try to reduce the blood loss during surgery.

The aim of surgery was to reduce as much as possible the thickness of the scalp infiltration, create 2 large biparietal flaps leaving a small strip of bone on the midline to protect the sagittal sinus, and offer structural support for the titanium meshes to use as temporary cranioplasty.

\section{First Surgery}

With the patient prone, a biparietal skin incision was performed to completely expose the tumor, which diffusely infiltrated the soft tissue of the scalp and underlying bone. Elevation of the scalp was difficult because of extreme bleeding and the thickness of the infiltrated scalp. The diploe appeared greatly thickened. And despite em- 

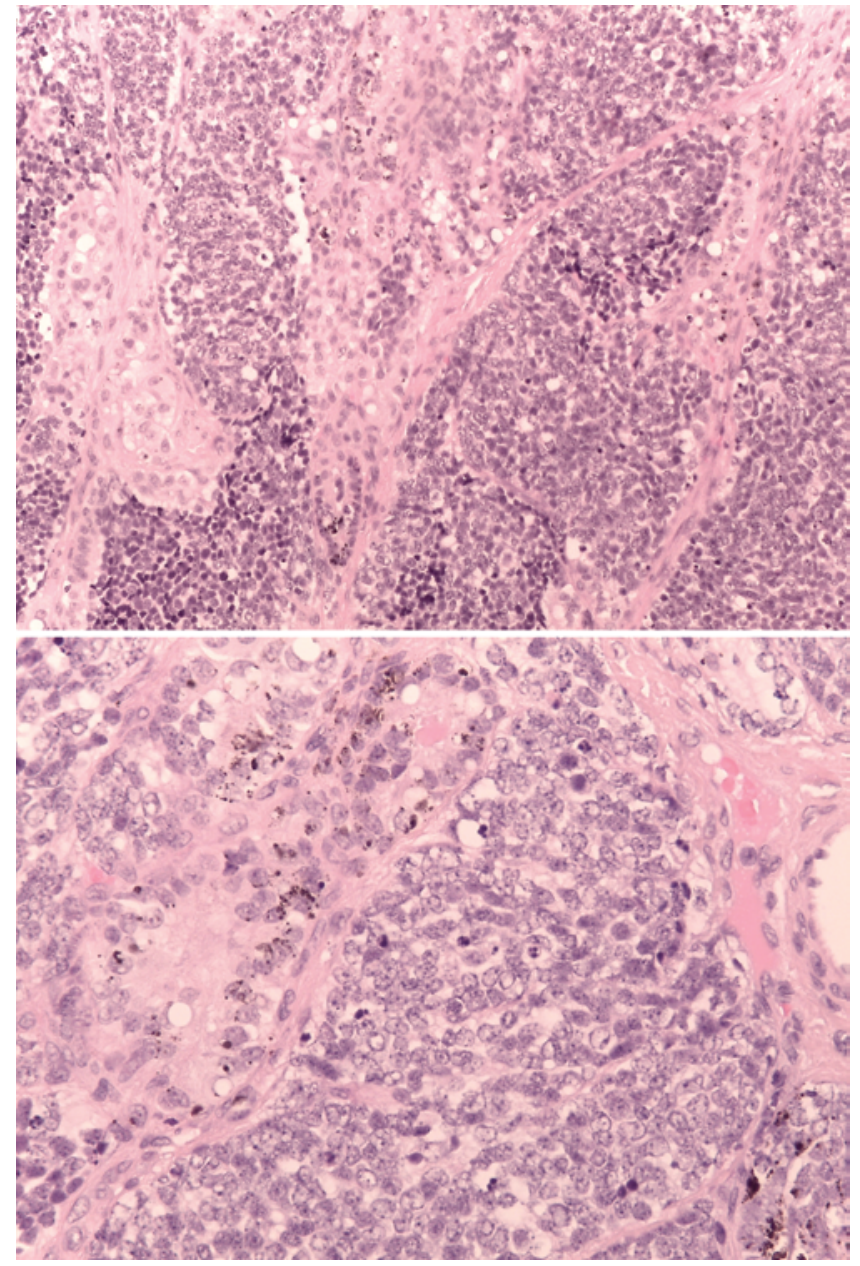

FIG. 3. Upper: Biphasic microscopic pattern. Lower: Epithelioid cells contain intracellular melanin granules. H \& E, $\times 200$ (upper) and $\times 400$ (lower). Figure is available in color online only.

bolization, the bony tumor was very hemorrhagic. The tumor tissue infiltrating the soft tissue was removed with the help of monopolar coagulation. The first necessary step in removing the thickened bone was to reduce the thickness of the parietal bone. The first flap was designed on the left side. Four bur holes $2 \mathrm{~cm}$ deep were made without reaching the dura: 2 parasagittal and 2 at the level of the temporal line. An oscillating saw was used to outline the flap, but hammer and chisel were used to split the parietal bone because of the severe blood loss induced by the use of the oscillating saw. After splitting the parietal bone, the 4 bur holes were continued in depth to reach the dural plane. The bone flap was completed with a bone rongeur parasagittally and with a high-speed drill using a 3-cm-deep footed attachment for laminotomy (Medtronic Inc.) on the other 3 sides. The whole parietal bone was removed, and the exposed infiltrated dura was replaced with a large nonresorbable duraplasty (Fig. 5A). At this point it became necessary to reduce the thickness of the bone on the midline and contralateral parietal bone (measured at 6 $\mathrm{cm}$ on the midline). Considering the very significant blood loss induced by the use of the oscillating saw whose $4-\mathrm{cm}$ blade allowed only piecemeal removal of bone fragments,

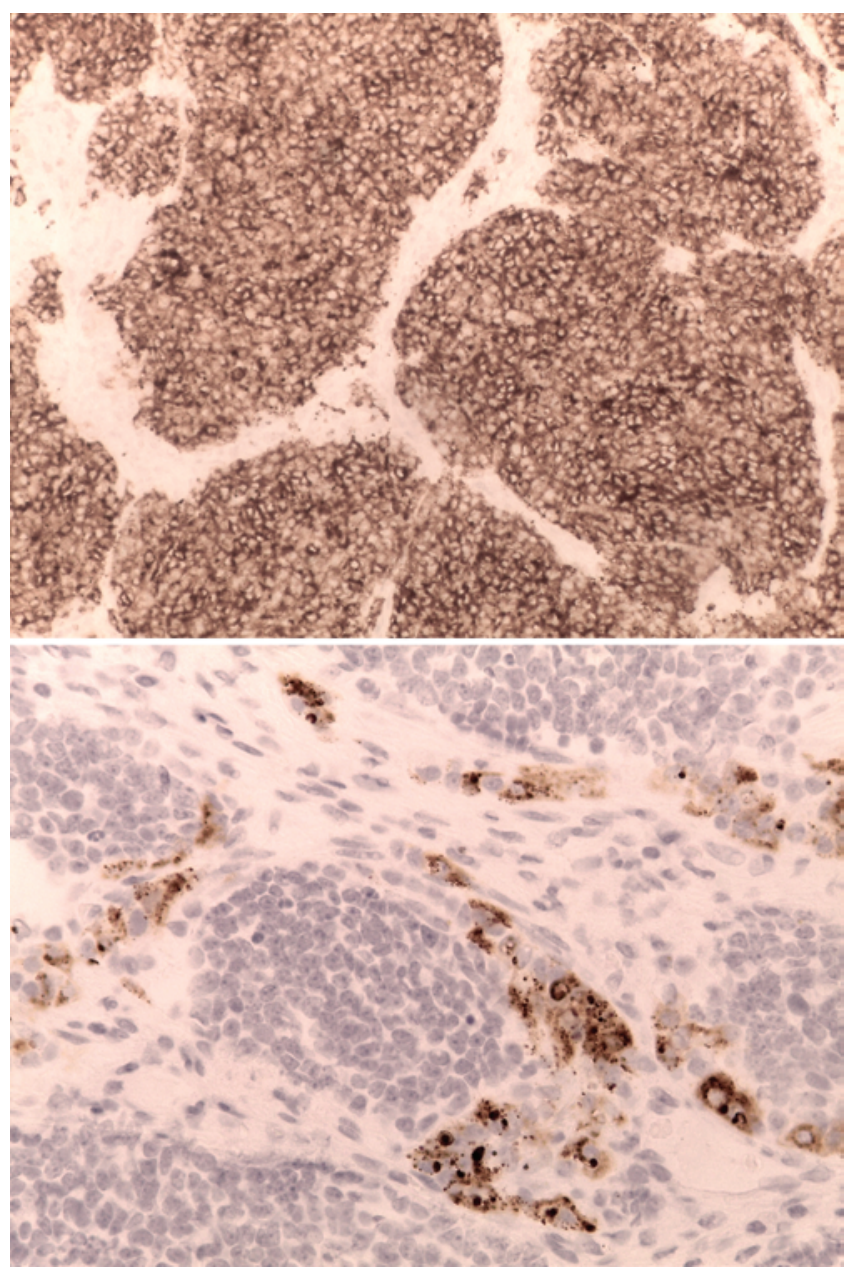

FIG. 4. Immunostaining for synaptophysin (upper) in a poorly differentiated cell and for HMB-45 (lower) in large cells. Original magnification $\times 200$ (upper) and $\times 400$ (lower). Figure is available in color online only.

we decided to split en bloc the remaining occipitoparietal bone using the Gigli saw, which proved incredibly effective in rapidly reducing the remaining bone uniformly to $<1-\mathrm{cm}$ thickness with acceptable blood loss (Fig. 5B). Because severe bleeding throughout the procedure led to hemodynamic instability of the patient, the bone tumor was only partially removed and the contralateral side with the suboccipital component down to the foramen magnum was left for the second procedure. The postoperative course was uneventful.

\section{Second Surgery}

The second surgery was planned for 1 month later, following a second session of endovascular embolization. At this time the tumor was almost totally removed, with complete craniectomy of the occipital bone of the posterior fossa through a large opening of the occipital foramen (Fig. $5 \mathrm{C})$. Because of the significant devascularization achieved with the first surgery and the 2 endovascular procedures, it was much easier to control blood loss during the second surgery while obtaining gross-total removal of the bone component and a drastic reduction of the infiltrative part at the level of the soft tissues of the scalp and neck. Only 

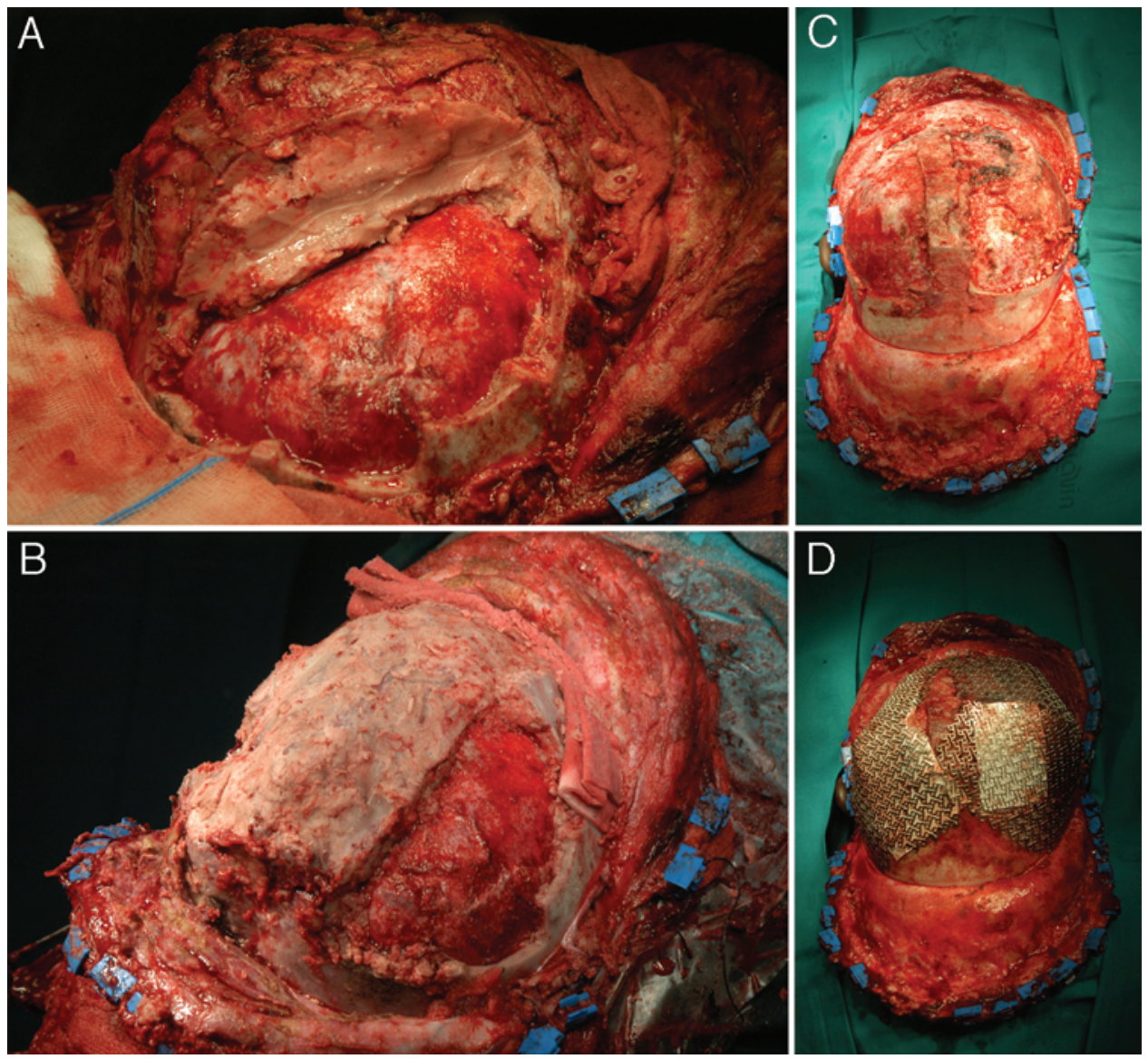

FIG. 5. A: First cranial procedure with the patient prone. A large occipitoparietal flap has been elevated on the left side. Calvarial thickness was measured at approximately $6 \mathrm{~cm}$ on the midline. B: In the same procedure, the thickness of the calvaria has been reduced to approximately $1 \mathrm{~cm}$ with a high-speed drill and Gigli saw. C: Second cranial procedure. Both parietal bones have been removed, and a large part of the occipital bone has been removed with foramen magnum decompression. A bone ridge $1 \mathrm{~cm}$ large and $5 \mathrm{~mm}$ thick has been left on the midline to protect the sagittal sinus and to offer support for titanium screws for calvarial reconstruction (D). Figure is available in color online only.

a very small portion on the midline bone, tightly adhering to the dura and superior sagittal sinus, was left in place to reduce the risk of bleeding. The bone defect was filled with 2 large titanium meshes fixed to the bone with screws (Fig. 5D).

\section{Second Postoperative Course}

Pathological findings and immunohistochemical studies confirmed the diagnosis of a primitive malignant lesion with a final diagnosis of neuroectodermal melanotic tumor. Immunohistochemical studies showed positivity for synaptophysin, cytokeratin (EMA1+EMA3), and HMB-45, as well as negativity for S-100 and glial fibrillary acidic protein.

After surgery MRI revealed tumor excision with the persistence of abnormal contrast enhancement of bone residue in the occipital region and persistence of an area of high signal intensity on T2 and FLAIR sequences within the left cerebellar hemisphere, which showed nuanced contrast enhancement (Fig. 2I and J). A CT scan showed good tridimensional reconstruction of the large area of the craniectomy (Fig. 6).

A PET-CT scan showed hyperaccumulation at the level of the left lateral cervical region; therefore, a new lymph node biopsy was performed at the left lateral cervical level, but it was negative for tumor tissue.

In view of the small residual tumor and the persistence of an area of contrast enhancement in the cerebellum, we decided to perform another cycle of chemotherapy using
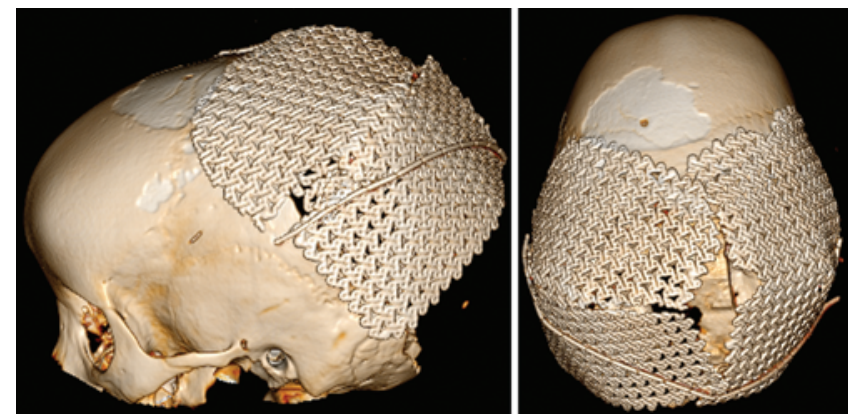

FIG. 6. Three-dimensional reconstructions of head CT following tumor removal. Note that both parietal bones and the occipital bone have been almost completely removed with foramen magnum decompression and reconstructed with titanium meshes. Figure is available in color online only. 
the same preoperative therapeutic protocol, followed by radiotherapy. However, after 2 cycles of chemotherapy, the boy's relatives refused further therapies including the scheduled radiotherapy and decided to take the child back to his country, after 18 months in Italy. Through a humanitarian organization that continued the child's follow-up and regularly sent pictures through social networks, we knew that the boy had local recurrence of the disease that rapidly grew and caused his death 6 months after the end of all treatments.

\section{Discussion}

Melanotic neuroectodermal tumor of infancy is a rare, mainly benign, pigmented lesion of neural crest origin, usually affecting infants in the 1 st year of life. ${ }^{1,2,7,15,18}$ Only $9 \%$ of cases are diagnosed after the age of 12 months. The tumor usually arises in the anterior maxillary alveolar ridge. Only a few cases occur in the neurocranium. The lesion often grows rapidly and generates a swelling or tumescence of the scalp, leading to local skull deformation, which is cosmetically obvious to parents of the infant.

Microscopic examination demonstrates a typical biphasic cell population composed of collections of larger, melanin-containing epithelial-like cells and smaller, round primitive neuroectodermal cells. Immunohistochemical studies are a great help in the diagnosis of MNTI: they are usually positive for cytokeratin, vimentin, epithelial membrane antigen, HMB-45, glial fibrillary acidic protein, and neuron-specific enolase. It is unclear which of these cell populations represents the main proliferative component of the tumor. Whereas immunohistochemistry indicates that expression of cell cycle proteins is restricted to the melanocytic cells, ${ }^{3}$ in cases in which MNTI has exhibited malignant behavior and metastasis, the dominant cell type has appeared neuroblastic.

Blood tests show high serum levels of catecholamines and increased urinary excretion of VMA and HVA, which underscores the close relationship with other tumors of neural crest origin. ${ }^{10,19}$ The neural crest origin for MNTI was independently proposed by Misugi et al., ${ }^{16}$ on the basis of electron microscopy examination of tumor, and Borello and Gorlin, ${ }^{3}$ who also observed that the high urinary levels of VMA, the main end-stage metabolite of catecholamines, returned to normal in a patient after tumor had been excised. Ultrastructural features, including characteristic melanin granules and modified tight junctions that support the view that MNTI is derived from the neural crest, were also identified by electron microscopy studies. ${ }^{5}$

The Raf-MEK-ERK mitogen-activated protein kinase pathway is activated by growth factors, hormones, and cytokines and regulates proliferation, differentiation, survival, senescence, and migration of cells. The most common $B R A F$ oncogenic mutation detected in human cancer is characterized by a $\mathrm{T}>\mathrm{A}$ transversion leading to a valine (V) to glutamic acid (E) amino acid substitution: V600E. This mutation in melanoma changed the pathological comprehension of the tumor, and new treatment options emerged from these findings. Given MNTI similarities with melanoma, researchers recently proposed a role of
BRAFV600E mutation in the pathogenesis of MNTI, providing new insights for personalized treatment. ${ }^{9}$

Approximately 437 cases of MNTI have been reported in the world literature since Krompecher first described it in 1918.,6,13 A review of the literature for the period 1977-2008 produced a total of 43 cases of MNTI located in the skull. According to Derache et al., ${ }^{6}$ skull localizations account for $15.6 \%$ of the 437 cases available in the literature. The tumor usually occurs at the junction of the skull bones. Analysis of the anatomical location of MNTI revealed 9 cases occurring in the anterior fontanelle, 5 in the orbit, 1 in the lambdoid suture, 14 in the occipital bone, 9 in the temporal bone, and 4 in the frontal bone; in 1 case the exact location in the skull was not reported.6.15

Melanotic neuroectodermal tumors of infancy are usually benign lesions. Complete surgical excision is the gold standard of treatment for lesions of the skull. Whenever bone is involved, wide tumor excision with a tumor-free margin is recommended. Surgery is usually simple and curative; however, some lesions raise particular surgical difficulties, especially when located along the cranial midline with infiltration of the dura of the dural sinuses, within cranial base bone, and with significant intracranial extension..$^{20}$

Postsurgery recurrence is reported in $28.3 \%$ of cases, mostly noticed between the 5th month and the 2nd year, and is attributable to inadequate excision, dissemination of neoplastic cells during surgery, or multicentricity. ${ }^{6}$ This rate of unfavorable evolution is probably overestimated given the fact that benign straightforward cases are less frequently the object of publication than cases having an unfavorable outcome..$^{14,16}$

In the literature malignancy in melanotic progonoma of the skull is reported in 6.6\% of cases. Malignant MNTI tends to have metastases in regional lymph nodes, liver, and bone marrow and spreads rapidly through the central nervous system and is associated with a high mortality rate. These patients have unusually high serum levels of catecholamines, which after surgery and chemotherapy may show a rapid decrease.

Malignant MNTI is addressed with surgery, which can be combined with other modes of treatment like chemotherapy alone or together with radiotherapy. Chemotherapy in combination with resection to treat primary or residual tumor has been described in 19 cases in the literature. ${ }^{6}$ It has proved to be effective in 11 cases, inducing complete regression ( 2 cases) or partial regression ( 9 cases), and ineffective in 8 cases for lack of reduction (3 cases) or tumor progression (5 cases). Chemotherapy was used following radical removal in 5 cases, with no recurrence in any of the cases. The drugs most frequently used are cyclophosphamide, vincristine, doxorubicin, etoposide, and carboplatin . This choice reflects the fact that one cellular population shows neuroblastic markers, but in fact there is no agreement on the preferred protocol in the literature.

The aim of the pharmacological schedule used in our patient was to obtain the quickest and most effective adjuvant result, considering the dramatic clinical features at presentation and the child's significant pain. The treatment was rapidly effective, both on the primary cranial lesion and on the secondary cervical bilateral involvement. Con- 
sidering the good response, regional involvement limited to the cervical lymph nodes, and the lack of other secondary dissemination, we decided to perform a staged surgery to achieve complete removal of the tumor, which is considered the gold standard of treatment. It was possible to anticipate the good response to chemotherapeutics commonly used in neuroblastoma because of the high level of VMA and HVA in our patient.

As known, MNTI histology shows 2 distinct populations of cells that form nests or alveolar structures: 1) large, polygonal epithelial-like cells containing melanin and resembling melanocytes, and 2) small, round neuroblast-like cells. Given the similarities of MNTI with melanoma, we planned to perform additional analyses, such as $B R A F$ oncogenic mutation to consider potential target therapy; unfortunately, however, the family refused additional intervention or analyses.

Radiotherapy combined with resection has also been reported in the literature in 9 cases, but its results remain highly uncertain. ${ }^{6}$

In our case chemotherapy was administered preoperatively because upfront radical resection was impossible and the general conditions of the child were extremely compromised. Chemotherapy allowed a rapid decrease in tumor volume and significant improvement of local and general conditions. After surgery it was administered to prevent dissemination of neoplastic cells because of incomplete resection of the midline tumor mass and in view of the planned radiotherapy, which was unfortunately refused by the parents.

Literature comparisons of the histological aspects of tumor before and after chemotherapy have shown a strong reduction in the neuroblast-like cells component. Since this component is regarded as the aggressive part of the tumor and responsible for the metastatic lesions in malignant cases, it seems important to emphasize the strong decrease in these cells in the postchemotherapy specimens. It also seems reasonable to proceed with preoperative chemotherapy in the hope of improving the surgical results and decreasing the risk of local recurrence or metastasis.

\section{Conclusions}

In accordance with previously reported cases in the literature as well as our own experience, no precise guidelines on the best treatment for this tumor type can be outlined. In smaller, more accessible cases of MNTI, radical tumor removal alone is certainly the best treatment in most cases. In cases of high-risk surgery for very large lesions or in cases with a high risk of cosmetically compromising procedures in the facial region, preoperative neuroblastoma-like chemotherapy should be considered as a useful step in the therapeutic plan, with the aim of reducing tumor volume and intraoperative blood loss and allowing better technical conditions for surgical removal. Chemotherapy in combination with resection seems to be a reasonable therapeutic option with the aim of improving surgical results, reducing surgical risks, and minimizing the risk of local recurrence or metastasis. The behavior of malignant MNTI is unpredictable; therefore, aggressive treatment is justified in cases of metastatic tumors at pre- sentation. In the future, personalized target therapy may play a role in maintenance and consolidation.

\section{Acknowledgments}

We gratefully acknowledge Dr. Vittoria Donofrio for revision of the histological specimens and supervision of the pathological implications of this case report.

\section{References}

1. Agarwal P, Agarwal V, Raina VK: Melanotic neuroectodermal tumour of infancy: case report of an unusual tumour. Indian J Plast Surg 36:123-125, 2003

2. Antunes ACM, Freitas RM, Oliveira PP, Rebouças RG: Melanotic neuroectodermal tumor of infancy: case report. Arq Neuropsiquiatr 63 (3A):670-672, 2005

3. Borello KD, Gorlin RJ: Melanotic neuroectodermal tumor of infancy-a neoplasm of neural crest origin. Report of a case associated with high urinary excretion of vanilmandelic acid. Cancer 19:196-206, 1966

4. Carpenter BF, Jimenez C, Robb IA: Melanotic neuroectodermal tumor of infancy. Pediatr Pathol 3:227-244, 1985

5. Cutler LS, Chaudhry AP, Topazian R: Melanotic neuroectodermal tumor of infancy: an ultrastructural study, literature review, and reevaluation. Cancer 48:257-270, 1981

6. Derache AF, Rocourt N, Delattre C, Vinchon M, Orbach $\mathrm{D}$, Leblond P: Les tumeurs neuroectodermiques mélanotiques infantiles: état actuel des connaissances. Bull Cancer 101:626-636, 2014

7. Gaiger de Oliveira M, Thompson LD, Chaves AC, Rados PV, da Silva Lauxen I, Filho MS: Management of melanotic neuroectodermal tumor of infancy. Ann Diagn Pathol 8:207-212, 2004

8. George JC, Edwards MK, Jakacki RI, Kho-Duffin J: Melanotic neuroectodermal tumor of infancy. AJNR Am J Neuroradiol 16:1273-1275, 1995

9. Gomes CC, Diniz MG, de Menezes GH, Castro WH, Gomez RS: BRAFV600E mutation in melanotic neuroectodermal tumor of infancy: toward personalized medicine? Pediatrics 136:e267-e269, 2015

10. Hoshino S, Takahashi H, Shimura T, Nakazawa S, Naito Z, Asano G: Melanotic neuroectodermal tumor of infancy in the skull associated with high serum levels of catecholamine. Case report. J Neurosurg 80:919-924, 1994

11. Johnson RE, Scheithauer BW, Dahlin DC: Melanotic neuroectodermal tumor of infancy. A review of seven cases. Cancer 52:661-666, 1983

12. Kaya S, Tinaztepe B: Melanotic progonoma of the maxilla (report of a successfully treated case and a brief review of the literature). Turk J Pediatr 24:183-188, 1982

13. Krompecher E: Zur Histogenese und Morphologie der Adamantinome und sonstiger Kiefergeschwulste. Beitr Pathol Anat Allg Patho 164:165-197, 1918

14. Kruse-Lösler B, Gaertner C, Bürger H, Seper L, Joos U, Kleinheinz J: Melanotic neuroectodermal tumor of infancy: systematic review of the literature and presentation of a case. Oral Surg Oral Med Oral Pathol Oral Radiol Endod 102:204-216, 2006

15. Lambropoulos V, Neofytou A, Sfougaris D, Mouravas V, Petropoulos A: Melanotic neuroectodermal tumor of infancy (MNT1) arising in the skull. Short review of two cases. Acta Neurochir (Wien) 152:869-875, 2010

16. Misugi K, Okajima H, Newton WA Jr, Kmetz DR, Delorimier AA: Mediastinal origin of a melanotic progonoma or retinal anlage tumor. Cancer 18:477-484, 1965

17. Mosby EL, Lowe MW, Cobb CM, Ennis RL: Melanotic neuroectodermal tumor of infancy: review of the literature and report of a case. J Oral Maxillofac Surg 50:886-894, 1992 
18. Omodaka S, Saito R, Kumabe T, Kawagishi J, Jokura H, Sonoda Y, et al: Melanotic neuroectodermal tumor of the brain recurring 12 years after complete remission: case report.

Brain Tumor Pathol 27:51-57, 2010

19. Pettinato G, Manivel JC, d'Amore ES, Jaszcz W, Gorlin RJ: Melanotic neuroectodermal tumor of infancy. A reexamination of a histogenetic problem based on immunohistochemical, flow cytometric, and ultrastructural study of 10 cases. Am J Surg Pathol 15:233-245, 1991

20. Pierre-Kahn A, Cinalli G, Lellouch-Tubiana A, Villarejo FJ, Sainte-Rose C, Pfister A, et al: Melanotic neuroectodermal tumor of the skull and meninges in infancy. Pediatr Neurosurg 18:6-15, 1992

\section{Disclosures}

The authors report no conflict of interest concerning the materials or methods used in this study or the findings specified in this paper.

\section{Author Contributions}

Conception and design: Cinalli. Acquisition of data: Nicosia, Cascone, Errico. Analysis and interpretation of data: Cinalli, Spennato. Drafting the article: Nicosia. Critically revising the article: Cinalli, Spennato, Quaglietta, Muto. Reviewed submitted version of manuscript: Cinalli. Approved the final version of the manuscript on behalf of all authors: Cinalli. Administrative/technical/ material support: Aliberti, Cascone, Quaglietta, Errico, Muto, Ionna. Study supervision: Cinalli, Ionna.

\section{Supplemental Information}

Current Affiliations

Dr. Nicosia: Pineta Grande Hospital, Castel Volturno CE, Italy.

\section{Correspondence}

Giuseppe Cinalli, Department of Pediatric Neurosurgery, Santobono-Pausilipon Children's Hospital, Via Mario Fiore n. 6, 80129 Naples, Italy. email: giuseppe.cinalli@gmail.com. 